\title{
Abordaje farmacológico de las recaídas en las adicciones
}

\author{
Alamo, C.; López-Muñoz, F.; Cuenca, E. \\ Departamento de Farmacología, Facultad de Medicina, Universidad de Alcalá, Madrid. \\ Enviar correspondencia a: \\ Prof. Cecilio Alamo, C/ Julio Camba 7. 28028 Madrid.
}

\section{RESUMEN}

La adicción es una enfermedad crónica, que requiere tratamiento durante la mayor parte de la vida del paciente y como tal, las recaídas y el incumplimiento terapéutico son hechos habituales. En las dos últimas décadas se ha avanzado notablemente en el conocimiento de las bases neuropsicobiológicas y farmacológicas de las adicciones, lo que ha permitido concluir que estamos ante una patología tratable. En la actualidad, se acepta que la fase más importante en la farmacoterapia de la dependencia es la que la que corresponde a la prevención de las recaídas. En este sentido, los antagonistas de los receptores opiáceos, en especial la naltrexona, constituye el avance más importante en esta fase preventiva. Su utilidad se ha demostrado en la prevención de la recaída a opiáceos y, más recientemente, en pacientes alcohólicos. De hecho, la naltrexona reduce en un $50 \%$ la tasa de recaídas y los individuos permanecen más tiempo abstinentes. Otros agentes utilizados en este campo son el acamprosato y los fármacos moduladores de la transmisión serotoninérgica, como la buspirona o los inhibidores selectivos de la recaptación de serotonina, fluoxetina y citalopram. Desafortunadamente, para otras dependencias (cocaína, cánnabis, anfetaminas, éxtasis, etc.) no existe un abordaje farmacológico específico, si bien se han empleado algunos de los fármacos comentados, así como antagonistas dopaminérgicos, con resultados poco significativos.

Palabras Clave: Adicciones, Recaídas, Farmacoterapia.

\section{SUMMARY}

The addiction is a chronic disease which requires treatment during most of the patient's life and as a consequence, the relapses and the therapeutic noncompliance are very common facts. In the last two decades, there have been remarkable advances in the knowledge of neuropsychobiological and pharmachological bases on the addiction field, which leads us to the conclusion that we are in front of a pathology susceptible of treatment. At present, it is accepted that the most important phase in the dependence therapy is the one corresponding the relapse prevention. In this sense, the opioid receptors antagonists, especially naltrexone, constitute the most important advance in this preventive phase. Its efficacy has been demonstrated in the prevention of the opiate relapse and, more recently, in alcoholic patients. In fact, the naltrexone reduces in a $50 \%$ the relapses and the patients remain abstinent for a longer time. Another agents of this group used are the acamprosate and the serotoninergic transmission modulating drugs, such as buspirone and the SSRIs (selective serotonine reuptake inhibitors), fluoxetine and citalopram. Unfortunately, for other dependences (cocaine, cannabis, anfetamines, ecstasy, etc.) there not exist an specific pharmacological treatment; however some of the above mentioned substances have been used, as well as dopaminergic antagonists, with poor results.

Key Words: Addictions, Relapses, Pharmacotherapy.

\section{INTRODUCCION}

$\mathbf{L}$ as adicciones y sus consecuencias socio-sanitarias constituyen un problema de una enorme dimensión. Pero, si además incluimos drogas de uso legal, como alcohol o nicotina, la dependencia constituye el enemigo sanitario número uno. En España, se calcula, a título orientativo, la existencia de alre- dedor de 2 millones de personas que abusan sistemáticamente del alcohol, reconociendo el $90 \%$ de la población haberlo tomado alguna vez. El 70\% de los españoles, con edades comprendidas entre 15 y 65 años, admiten haber fumado, y un 25\% son fumadores habituales. Existen casi 200.000 adictos a los opiáceos, aunque su consumo no evidencia una tendencia incremental. Entre las sustancias psicoestimulantes destaca la cocaína, consumida por un $3,2 \%$ de los 
españoles, y en situación de crecimiento, los alucinógenos, consumidos por el 2,6\%, y las anfetaminas por el $2,5 \%$. El éxtasis es consumido por el 6,2\% de los jóvenes entre 19 y 28 años y aproximadamente el $4 \%$ de los menores de 18 años admiten haberlo consumido. En nuestro medio, se calcula la existencia de más de un millón de fumadores de cannabis, al menos esporádicos, y el $4 \%$ reconocen fumarlo habitualmente, cifra que asciende hasta un 9,4\% entre los 19 y 28 años. Además, existe un número indeterminado de personas que abusan de diversos fármacos (Cabrera, 1998; Nuñez y cols., 1998).

Pero si la incidencia de las adicciones es elevada, la repercusión sanitaria lo es, aún, en mayor medida. Así, en EE.UU., se ha calculado que el $40 \%$ de los ingresos hospitalarios y el $25 \%$ de los fallecimientos, a lo largo de cada año, se deben, de forma directa o indirecta, al consumo de tabaco. Alrededor del $5 \%$ de todas las muertes se deben al consumo de heroína o cocaína. En la mitad de las muertes por accidente de tráfico existe la presencia de algún intoxicado responsable. En el $50 \%$ de los casos de violencia doméstica está presente la adicción a alguna droga ilícita o alcohol. Además, la comorbilidad de abuso de sustancias con psicopatología, lo que se ha dado en llamar trastorno dual, oscila, según las estadísticas, entre un 20 y un $90 \%$ de los casos (A.P.A., 1995). Finalmente, los problemas sanitarios infecciosos (hepatitis, SIDA, abscesos, etc.), ocasionados por falta de medidas higiénicas en la administración de drogas suelen ser la norma (Gerada y Ashworth, 1997). En definitiva, entre el 10 y el $16 \%$ de los pacientes que visitan al generalista padecen problemas relacionados con la dependencia y abuso de sustancias (Weaver y cols., 1999).

Sin embargo, pese a la frecuencia y trascendencia del problema de las dependencias, los conocimientos y habilidades del personal sanitario y la dedicación que la docencia oficial (Leshner, 1999) dedica a estos aspectos no siempre están en consonancia con la importancia de esta patología. Una posible explicación de este desinterés académico puede deberse a que, tradicionalmente, las dependencias han sido consideradas, no solo a nivel popular, como "fallos morales" o como conductas malintencionadas, pensándose que la mayoría de las personas que abusan de sustancias ilícitas son marginales, sin trabajo, concentrados en las zonas más empobrecidas de las ciudades. Evidentemente el problema en esta franja social existe, pero no en exclusiva. En efecto, un estudio realizado durante el año 1997 por el NHSDA (National Household Survey on Drug Abuse) demostró que el $70 \%$ de los 6,3 millones de personas, con edades comprendidas entre 18 y 49 años, que admitieron haber consumido sustancias ilícitas en el último mes, tenían un empleo fijo. Por tanto, el mito que habla de la droga como un problema que afecta únicamente a marginales debe revisarse (Marwick, 1999).
Ante esta forma de pensar, todos hacemos esfuerzos para reconsiderar el problema de las adicciones como una enfermedad del cerebro (Kosten, 1998), en la que existe un claro nexo entre alteraciones conductuales y neurobiológicas. Una mejor formación en estos procesos permitirá a los clínicos, en la actualidad más familiarizados con estas disciplinas, integrar estos conocimientos y conseguir un plan terapéutico que contemple aspectos psicoterapéuticos, farmacológicos (Iglesias y cols., 1992) y sociales, todos ellos apoyados en las nuevas herramientas tecnológicas.

\section{CONCEPTUALIZACIÓN DE LAS ADICCIONES COMO ENFERMEDADES CRÓNICAS}

Precisamente, desde la perspectiva educativa, hay que tener presente el concepto de la adicción como una enfermedad crónica, al igual que el asma, la diabetes, la colitis ulcerosa, la esclerosis múltiple o la hipertensión. Todos estos procesos requieren tratamiento durante la mayor parte de la vida del paciente y, como tal, las recaídas y el incumplimiento de las pautas de medicación son hechos habituales. Sin embargo, en la adicción, en la mayoría de ocasiones, los esfuerzos se centran en la corrección del problema agudo. Por ello, cuando el paciente recae, al igual que sucedería en las patologías somáticas crónicas si suspendiéramos el tratamiento, la intervención se considera un fracaso. Este concepto ha hecho que exista una extendida percepción de la ineficacia de los tratamientos de las dependencias, lo que lleva a muchos médicos a renunciar a ellos. Sin embargo, la dificultad de tratamiento de las adicciones es compartida por otras patologías crónicas y no por ello se renuncia al tratamiento. Así, en la diabetes, el porcentaje de pacientes que cumplen la dieta recomendada es tan solo del $30 \%$ y la cumplimentación de la medicación se realiza sólo por el $40 \%$ de los mismos. En estas circunstancias no es sorprendente que las recaídas, a lo largo de un año de seguimiento sean del orden del $40 \%$. Asimismo, las recaídas son habituales en la hipertensión arterial, un 55\% a lo largo del año, y casi la norma en el asma bronquial, con porcentajes de recaídas del orden del 70\% (O’Brien y McLellan, 1996). En este sentido, es importante destacar la preocupación existente entre los profesionales sanitarios de países desarrollados por la importancia de las patologías crónicas como primera causa de mortalidad y la creciente dificultad de realizar en ellas un control sanitario adecuado. Así, el 59\% de los diabéticos incluidos en el Plan de Salud HEDIS (Health Plan Employer Data and Information Set) durante 1998 no tuvieron un examen oftálmico en el ultimo año, el $46 \%$ de los pacientes tratados con antidepresivos no estuvieron controlados correctamente y al $41 \%$ de los pacientes 
ingresados en un hospital por algún evento cardiovascular no se les controló el colesterol tras su alta (Davis y cols., 2000). Por tanto, la insatisfacción por el control de las patologías crónicas no afecta sólo al problema de la dependencia, ni es un problema único de nuestro entorno. De hecho, en lo referente a las adicciones los éxitos terapéuticos pueden considerarse relevantes en algunos casos y menos importantes en otros cuadros. En este sentido, el rango de éxito terapéuti$\mathrm{co}$, en un periodo de 6 meses, fue del 50 al $80 \%$ en el caso de la dependencia a opiáceos, del 50 al $60 \%$ en la adicción a cocaína, del 40 al $70 \%$ en alcohólicos y del 20 al 40\% en la adicción a nicotina (Fig. 1) (O’Brien y McLellan, 1996). Por todo ello, en lo referente a las adicciones, el nihilismo terapéutico no parece estar justificado.

\section{FIGURA 1: Indices de éxito terapéutico, a 6 meses, en distintos trastornos por abuso de substancias.}

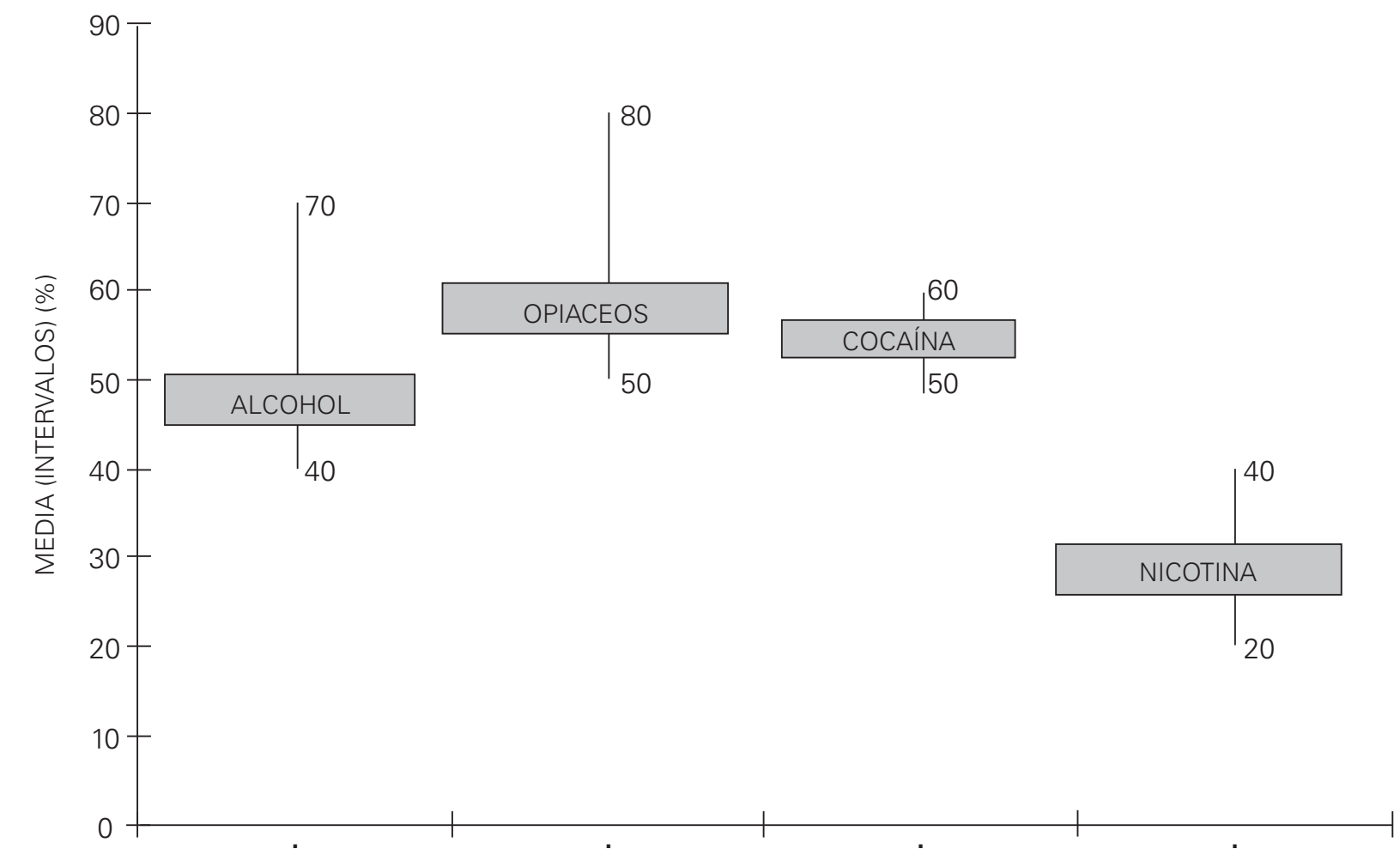

Modificada de O'Brien y McLellan (1996).

En efecto, en las dos últimas décadas hemos avanzado en el conocimiento de las bases neuropsicobiológicas y farmacológicas de las adicciones, lo que ha permitido acercarnos a la conclusión de que esta patología, con los problemas inherentes a cualquier entidad crónica, es tratable. Obviamente, no todas las dependencias responden a un mismo tratamiento, ni con la misma eficacia, ya que, si bien en esencia la adicción es una enfermedad cerebral, el fenómeno es más complejo, ya que está influenciado por una frecuente patología psiquiátrica comórbida (trastorno dual), así como por aspectos sociológicos y conductuales que incluyen los estímulos iniciales para el consumo de la droga. Por todo ello, el abordaje terapéutico de esta patología es mucho más eficaz si se realizan programas de tratamiento multidimensio- nales, que incluyan aspectos psicológicos, farmacológicos, médicos, sociales, legales, etc. (Leshner, 1999). Si estos programas son adaptados de forma individual a la medida de cada paciente, podremos conseguir reducir el consumo, la avidez ansiosa ("craving"), las recaídas y las consecuencias médico-sociales y legales de la dependencia. Sin embargo, con frecuencia se olvida que la adicción no termina cuando la droga desaparece del organismo (desintoxicación), ya que los procesos adaptativos provocados por el consumo permanecen, lo que lleva a la recidiva con un nuevo consumo de la droga (O'Brien y McLellan, 1996).

Desde una perspectiva puramente farmacológica, que debe tener siempre en consideración ser una pieza más de este abordaje multifactorial, podemos considerar tres ejes de tratamiento de las dependen- 
cias: terapia del síndrome de abstinencia, mantenimiento y prevención de las recaídas. Estas medidas complementarias deben ser adaptadas a cada sujeto. Así, un individuo puede tener que ser sometido a terapia de mantenimiento durante un periodo de tiempo indefinido, mientras que otros pueden beneficiarse de una terapia de prevención de recaídas, tras ser tratados del síndrome de abstinencia, sin necesidad de pasar por la fase de mantenimiento.

\section{EL PAPEL DEL “CRAVING"Y LOS "CUE" EN LAS RECAÍDAS}

En la actualidad, se acepta que la fase más importante en la farmacoterapia de la dependencia es la que corresponde a la prevención de las recaídas. La recaída puede definirse como la reanudación de una conducta de búsqueda y readministración de la droga, tras un periodo de abstinencia (Altman y cols., 1996).

Tanto el craving como el cue son términos de difícil definición, que se relacionan, en distinto grado, con la recaída. Para algunos expertos, el craving podría considerarse como un signo indicativo de que el adicto tiene un problema, la falta de la droga, y es un marcador cognitivo del inicio de una batalla real en el cerebro del adicto. Para Tiffany (1990), el proceso que controla el abuso de drogas y el que media el craving serían independientes. El craving constituiría un proceso cognitivo dedicado a impedir o ayudar, según el caso, a la ejecución automática de la conducta de abuso de droga. Una interpretación alternativa del craving es la que lo considera como una experiencia subjetiva de un fuerte o intenso deseo de experimentar las propiedades modificadoras del humor producidas por una droga. Es difícil asimilar este intenso deseo como algo psicopatológico, ya que se presenta, también con frecuencia, para estímulos no farmacológicos, que se consideran, habitualmente, poco perjudiciales y manejables. La CIE-10 y el DSM-IV enfocan el término como una urgencia incontrolable de consumir la droga y la perdida de control es un punto central en la descripción de la dependencia. En este concepto, por tanto, el craving se centraría en una hipótesis esencialmente motivacional. Sin embargo, esta aproximación motivacional no explica si el craving busca sólo los aspectos de refuerzo positivo de la droga o el deseo de evitar los aspectos negativos del síndrome de abstinencia (Altman y cols., 1996).

El craving depende de la clase de droga y probablemente de la personalidad básica y características inherentes del individuo. Desde una perspectiva clínica, se considera que las drogas que tienen la capacidad de inducir un craving más intenso son los opiáceos, los psicoestimulantes, el alcohol y la nicotina (Baker y cols., 1987).
La importancia de la implicación del craving en la adicción y dependencia es motivo de desacuerdo entre los clínicos, que lo consideran relevante, y los investigadores, que lo entienden como un epifenómeno conductual de poca trascendencia, al detectarse una doble disociación entre el nivel de craving expresado por los adictos y el trabajo realizado para acceder a la administración de la droga, en dependencia con la sustancia en juego (Foltin y Fischman, 1994). En este sentido, es necesario tener en consideración que los clínicos tratan con pacientes que pueden "exagerar" la sintomatología de su craving para conseguir que se les suministre ayuda o la propia droga, mientras que los investigadores estudian sujetos que pueden convivir, de mejor o peor forma, con su dependencia. En cualquier caso, unos y otros coinciden en la necesidad de investigar con mayor intensidad en el fenómeno del craving, aunque sólo se tratara de un fenómeno conductual (Altman y cols., 1996).

No siempre es necesario el craving para que se produzca la recaída, por lo que se pueden considerar ambos fenómenos relacionados, pero independientes. En efecto, muchos pacientes consultados señalan que muchos episodios de craving no precedieron a las recaídas, mientras que sólo una pequeña proporción de pacientes que recayeron señalaron, retrospectivamente, que presentaron craving antes de la recaída. Pese a ello, desde una perspectiva farmacológica, a los medicamentos que presentan un cierto nivel de eficacia en la prevención de las recaídas se les denomina, en ocasiones, agentes "anti-craving" (Spanagel y Zieglgänsberger, 1997), si bien el mecanismo exacto de este fenómeno, tanto desde una perspectiva experimental animal como clínica, no resulta fácil de estudiar.

La prevención de las recaídas es la clave para el tratamiento de todas las adicciones. Por tanto, debemos conocer no sólo los efectos de las drogas sobre el cerebro, sino también, y sobre todo, los efectos residuales que hacen que un adicto "limpio" de droga recaiga de forma compulsiva (O'Brien, 1997). Una forma actual de ver las adicciones indicaría que cada episodio de exposición a la droga activaría estructuras cerebrales específicas, dejando trazas en la memoria que persistirían durante mucho tiempo después de que la droga haya desaparecido del organismo. Dichos episodios estarían relacionados con factores o señales del entorno (personas, lugares, objetos, pensamientos), que adquieren la capacidad de activar idénticos, o complementarios, circuitos cerebrales, incluso en ausencia de la droga. Estos factores o señales del entorno son denominados en terminología inglesa "cue" (taco de billar), acepción que no tiene fácil traducción científica al castellano y que algunos autores llaman señales. Podemos, por tanto, entender los cue como señales que el sujeto relaciona con los efectos de la droga y que, en cierta medida, le inducen a la 
recaída por poner en marcha, de forma anticipatoria, circuitos cerebrales modificados por la pasada experiencia con la droga. Estas señales podrían, a título de ejemplo, ser personas (un encuentro con un compañero de adicción o con el suministrador de la droga), lugares (la visión del parque donde se administraba la droga o el bar donde tomaba alcohol), publicidad explícita o subliminal de sustancias de abuso, objetos (la visión de una jeringa o de una navaja, en el caso de dependencias a heroína o cocaína) o, incluso, pensamientos, estados de ánimo o estados patológicos del individuo.

Las recaídas han sido estudiadas desde diferentes perspectivas. Una aproximación conductual clásica (Wikler, 1973) escapa a los objetivos de este trabajo. Asimismo, otra aproximación al conocimiento de las recaídas y al de los efectos persistentes de las drogas tras su desaparición del organismo ha empleado las modernas técnicas de neuroimagen. Así, se ha podido detectar, con técnicas de Tomografía de Emisión de Positrones (PET) y de Resonancia Magnética (RMI), en adictos a cocaína, que las señales o cue, en ausencia de la administración de la sustancia, provocan una activación de circuitos cerebrales específicos (O'Brien, 1997).

\section{ASPECTOS BIOQUÍMICOS EN LOS FENÓMENOS DE RECAÍDA}

En las últimas décadas se ha avanzado, a nivel molecular, en el conocimiento de los cambios que producen la administración continuada de drogas (Alguacil y cols., 1991). En este sentido, entre los múltiples posibles substratos implicados en los mecanismos de la drogodependencia debemos también considerar aquéllos que están regulando la expresión génica (Nestler, 1994). En efecto, una explicación de un efecto que se manifiesta tras la suspensión de la administración continuada de una droga sugiere la existencia de cambios celulares diferidos que requieren la puesta en marcha de nuevos mecanismos en la bioquímica celular (Fig. 2), como, por ejemplo, modificaciones en la síntesis de receptores, péptidos, enzimas, etc. (Self y Eric, 1995). Estos cambios son, lógicamente, consecuencia de modificaciones en el programa genético de la célula.

FIGURA 2: Esquema representativo de las modificaciones bioquímicas intracelulares desencadenadas por la administración crónica de opiáceos, que originarían, finalmente, alteraciones en la expresión génica de la neurona.

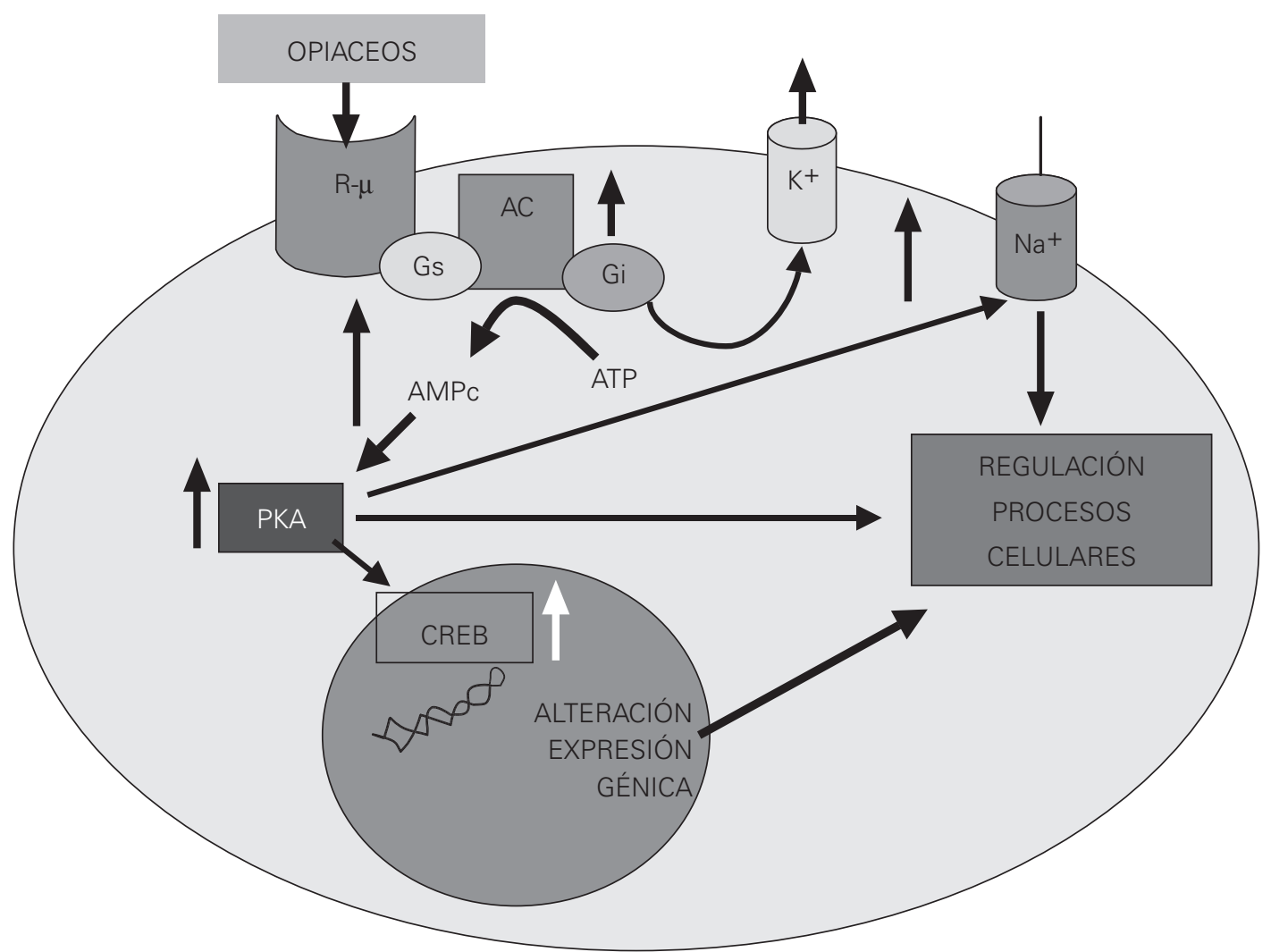

G (proteínas G); AC (adenilatociclasa); PKA (proteinkinasa A); CREB (cAMP response element binding protein). 
Existen dos tipos de evidencias que sugieren que la alteración en la expresión génica puede contribuir de forma importante a la drogodependencia. En primer lugar, varias proteínas dianas (proteínas G, proteínas de la vía de AMPc) identificadas en el sistema dopaminérgico mesolímbico son reguladas por drogas, y esta regulación se produce por medio del RNA mensajero. En segundo lugar, se conoce, desde hace bastantes años, que muchos hechos importantes de la adicción, tanto en el humano como en el animal de laboratorio, pueden persistir por un período prolongado de tiempo, incluso toda la vida, tras la discontinuación de la exposición a la droga. Estas consideraciones han estimulado a muchos investigadores a examinar directamente la regulación de la expresión génica por drogas de abuso.

Estos estudios se han enfocado hacia una serie de proteínas reguladoras nucleares, denominadas factores de transcripción, que se unen de forma específica a secuencias de DNA localizadas en las regiones promotoras de algunos genes y que, en realidad, aumentan o disminuyen la tasa de transcripción de dichos genes. Los factores de transcripción más conocidos son el CREB, el FOS y proteínas relacionadas (Widnell y cols., 1996).

En esta línea, se puede comprobar que las drogas que producen alteraciones en las vías intracelulares de mensajeros pueden provocar alteraciones en la función de los factores de transcripción, ya sea a través de una fosforilización directa de dichos factores, o a través de la regulación de su expresión (Fig. 2). Esta regulación de los factores de transcripción puede alterar la expresión de proteínas diana específicas, que serían responsables de los cambios adaptativos de la función celular que conducen a la adicción. Así, es importante detectar cambios que se producen como consecuencia de la administración de drogas a largo plazo. Es conocido que la administración crónica de opiáceos regula proteínas dependientes del AMPc, en el núcleo accumbens y en otras regiones cerebrales implicadas en las propiedades reforzadoras de los opiáceos (Nestler, 1994). Además, la adaptación de estas vías y núcleos puede contribuir a cambios en los mecanismos de refuerzo que caracterizan la adicción a opiáceos.

Se ha examinado el posible papel de los factores de transcripción CREB en la mediación de los efectos a largo plazo de los opiáceos en el núcleo accumbens, comprobándose que la administración crónica, aunque no la aguda, de morfina provocó una disminución de los niveles de la inmunorreactividad CREB en este núcleo. Este efecto fue específico, ya que no se observó en otras regiones cerebrales estudiadas. Estos resultados hablan a favor de un papel para el $C R E B$ en la autorregulación de la vía del AMPc en el SNC, así como en la mediación de algunos de los efectos de esta vía del núcleo accumbens (Widnell y cols., 1996). Estos datos tienen gran importancia, ya que demuestran una evidencia directa de la capacidad de la morfina para producir una adaptación de una proteína diana específica "in vivo", en una zona, el núcleo accumbens, de conocida significación funcional en el fenómeno de la drogodependencia (Alamo y cols., 1996).

Estos cambios en la transcripción génica, que ponen en marcha mecanismos de adaptación, están, sin duda, en la base de la dependencia y por tanto constituyen dianas de estudio para el futuro abordaje farmacológico de las recaídas.

\section{ABORDAJE FARMACOLÓGICO DE LAS RECAÍDAS}

Entre los objetivos para el abordaje farmacológico de las recaídas se incluyen medicamentos capaces de abolir o reducir la frecuencia e intensidad de las mismas. En este sentido, lo ideal es conseguir la ausencia total de recaídas o, lo que es lo mismo, la abstinencia total, lo que se acompañaría de un mejor pronóstico. Pero en la mayoría de los pacientes, la abstinencia absoluta es muy difícil, por lo que, para estos pacientes, una reducción en la frecuencia y severidad de las recaídas puede constituir un objetivo terapéutico aceptable (Conferencia de Consenso NIH, 1998).

Es muy importante para los clínicos y para los pacientes comprender la naturaleza crónica de esta patología y por ello debe establecerse, al igual que se hace en otras patologías crónicas somáticas, algún tipo de intervención tras una nueva recaída (Rubio y cols., 1992). Los pacientes deben reconocer las señales de peligro para evitar recaídas intensas tras la toma de pequeñas cantidades de alcohol o drogas. Pero si la recaída se produce, más que culpabilizar al paciente debemos intensificar el tratamiento (Weaver y cols., 1999).

En líneas generales, se considera que las alternativas farmacológicas para el tratamiento de las dependencias pueden encuadrarse en cuatro diferentes grupos: terapéutica de sustitución, terapia con antagonistas, agentes aversivos y fármacos que disminuyen la avidez (craving).

La terapia de sustitución se basa en la administración de un fármaco con propiedades similares a la droga ilícita con el fin de establecer una mejoría personal y social del paciente. El prototipo de este tipo de terapia es la metadona, que reduce el consumo ilícito y los problemas sanitarios y sociológicos secundarios a la administración intravenosa de heroína. En sentido estricto no podríamos hablar de la abolición de recaídas con este tipo de terapia, ya que el paciente está siendo tratado con una droga con idéntico mecanismo 
de acción y, por consiguiente, la recaída no debería producirse.

El ejemplo más característico de la terapia con antagonistas es el representado por la naltrexona en el tratamiento de la dependencia a heroína. En los adictos bajo tratamiento estabilizado con naltrexona, la administración de heroína no se acompaña de su característico efecto reforzador, cognitivo y conductual, ni de la subsiguiente búsqueda y avidez por conseguir nueva droga, ya que el adicto sabe que no le va a producir la acostumbrada recompensa. Sin embargo, el tratamiento de la adicción a la heroína con naltrexona presenta algunos problemas. El más importante es el alto grado de incumplimiento por parte de estos pacientes. Además, la duración del efecto de la naltrexona es corta, por lo que muchos pacientes suelen recaer, en lo que se ha dado por denominar las recaídas del fin de semana. Por otro lado, el establecimiento del tratamiento puede acompañarse de cierto grado de disforia y debe instaurarse gradualmente para evitar la producción de síndromes de abstinencia. Los mejores resultados se han obtenido en pacientes con una alta motivación (Best y cols., 1996). Por ello, los expertos parecen inclinarse por una mayor facilitación de la implantación de los programas de metadona como tratamiento de la dependencia a opiáceos (NIH, 1998), habiéndose demostrado una alta tasa de eficacia en la reducción de la administración intravenosa de heroína $y$, consecuentemente, una menor incidencia de morbimortalidad por VIH. Asimismo, estos programas disminuyen la tasa de criminalidad y de absentismo laboral (Leshner, 1999).

El uso de terapias aversivas ha sido ampliamente usado, en especial, para corregir el alcoholismo. En este sentido, se ha empleado sobre todo el disulfiram, cuyo nombre comercial "antabus" ha sido acuñado para definir la interacción indeseable de cualquier medicamento con el alcohol.

El disulfiram plantea un interesante dilema. Durante más de 50 años ha sido considerado por los clínicos como un agente de gran valor para evitar las recaídas. Sin embargo, los ensayos clínicos controlados indican una ambigua eficacia. En estos, se comprueba que el disulfiram reduce la frecuencia de ingesta de alcohol, pero no potencia las tasas de abstinencia. El control de la medicación mejora los resultados; sin embargo, este control solo se realizó en un estudio (Chick y cols., 1992), que no guardaba la metodología del "doble ciego".

Los implantes de disulfiram parecen aportar una garantía de permanencia del producto en el organismo. No obstante, en ninguno de los estudios realizados se ha comprobado el nivel plasmático de disulfiram. Además, la realización de una prueba de alcohol en los pacientes implantados con disulfiram no provocó el efecto "antabus". En resumen, de los estudios clínicos revisados se puede concluir que el disulfiram implantado reduce el número de días de bebida, si bien estos resultados no son siempre consistentes, pero no mejora otras medidas de consumo de alcohol. En definitiva, las terapias con disulfiram deberían emplearse con menor frecuencia, aunque pueden jugar aún algún papel en determinados pacientes (West, 1999).

Los fármacos que reducen la avidez (agentes anti-craving) y, a través de este mecanismo, reducen las recaídas son los que gozan de mayor interés desde una perspectiva de futuro. Estos agentes actuarían sobre mecanismos que no son substratos directos de la droga. Los agentes más estudiados encuadrados dentro de este grupo van fundamentalmente encaminados a prevenir las recaídas al alcohol. Entre éstos se encuentran los antagonistas opiáceos, naltrexona y nalmefene, el análogo de la homotaurina, acamprosato, y algunos agentes que actúan sobre mecanismos serotoninérgicos.

\section{LA TERAPÉUTICA "ANTICRAVING" COMO FUNDA- MENTO DE LA PREVENCIÓN DE RECAÍDAS: EL EJEMPLO REPRESENTATIVO DE LA DEPENDEN- CIA ALCOHÓLICA.}

Durante mucho tiempo se ha especulado sobre la relación existente entre alcoholismo y el sistema opioide. De hecho, a principios del siglo XX se recogía en el catalogo Sears una tintura de morfina para el tratamiento del alcoholismo, así como una solución alcohólica para el tratamiento de la adicción a morfina (Ulm y cols., 1995). En la actualidad, existen múltiples evidencias que sugieren que el sistema opioide forma parte de los neurocircuitos responsables del alcoholismo. De hecho, algunos estudios experimentales ponen de manifiesto que los antagonistas de receptores opioides, naloxona o naltrexona, disminuyen la autoadministración de etanol en diversas especies de animales de experimentación (Volpicelli y cols., 1997). Además, en la actualidad, la experiencia clínica ha permitido que la naltrexona se haya convertido en un tratamiento aprobado para el alcoholismo (Ochoa, 1998; Guardia, 1998).

Los mecanismos que intentan explicar la interacción entre el alcohol y el sistema opioide, siguiendo a Ulm y cols. (1995), se centran en diferentes aspectos:

Un primer mecanismo intentaría explicar la interacción a través del estímulo de receptores opioides por derivados metabólicos del alcohol. La condensación de acetaldehído con dopamina formaría derivados tetrahidroisoquinolínicos, capaces de estimular receptores opioides. Sin embargo, este mecanismo no parece, por si solo, explicar la interacción, ya que las concentraciones cerebrales alcanzadas de tetrahidroisoquinolinas son poco trascendentes. 
Una segunda propuesta de interacción vendría dada por la capacidad exhibida por el alcohol de modificar las propiedades de los receptores opioides. Si bien muchos de estos estudios, realizados tanto in vitro como in vivo, son equívocos, podría aceptarse que el etanol incrementa la densidad de receptores $m$ $y$, por otra parte, provoca un incremento marcado y selectivo del RNAm codificador de receptores opioides d, que se acompaña, además, de un aumento de la expresión de estos receptores.

La tercera posibilidad de interacción entre el sistema opioide y el alcohol se basa en la capacidad exhibida por el alcohol de modificar el contenido y la liberación de péptidos opioides endógenos, en diversas áreas y tejidos cerebrales. Así, se ha podido demostrar que la administración aguda de etanol provoca un incremento de los opioides plasmáticos, al igual que un incremento de los niveles de metencefalina y b-endorfina en hipotálamo, hipófisis y núcleo estriado. Estos opioides endógenos actúan preferentemente sobre receptores $\mathrm{m}$ y d, por lo que provocarían un refuerzo positivo en el animal de experimentación. Este fenómeno es más marcado en las ratas con preferencia genética por el alcohol (Alamo y cols., 1999).

En el humano, la relación de alcohol y sistema opioide ha sido asimismo estudiada por diferentes grupos de trabajo. En este sentido, se ha podido observar, en personas con factores de riesgo para el alcoholismo, que la ingestión de alcohol produce un incremento de b-endorfina en el plasma (Gianoulakis y cols., 1990). Además, se ha observado que los individuos con riesgo familiar de alcoholismo exhiben una mayor sensibilidad a la naloxona, lo que es indicativo de una menor actividad opioide a nivel hipotalámico.

La relación entre conducta adictiva alcohólica y sistema opioide se ha intentado explicar, fundamentalmente, mediante dos hipótesis: una que hablaría de un déficit opioide y otra justificada en un exceso opioide (Alamo y cols., 1999).

La hipótesis del "déficit" basal de funcionalismo opioide, se sustentaría en que el citado déficit sería compensado por la administración de etanol. Según esta hipótesis, el déficit sería el responsable de la avidez (craving) y el consumo de etanol sería inversamente proporcional al funcionalismo del sistema opioide endógeno. En este sentido, el alcohol, estimulando la liberación de endorfinas compensaría el déficit opioide. Esta compensación mantendría el refuerzo y el consumo.

La hipótesis del "exceso" atribuye la perdida del control a la existencia de un hiperfuncionalismo opioidérgico en el alcohólico, por el cual se aumenta la probabilidad de que el sujeto empiece a beber. Una vez iniciado el consumo, el alcohol incrementaría la función opioide, y por tanto el refuerzo, por lo que el suje- to perdería el control y seguiría consumiendo. En apoyo de esta hipótesis, estaría la observación de que la administración de dosis bajas de opioides provoca un fenómeno de cebado o encendido, que iniciaría el consumo de alcohol.

Ambas hipótesis tienen en común que el sistema opioide es el responsable del mantenimiento del consumo de alcohol (Fig. 3), por lo que la observación de la disminución de consumo por parte de antagonistas opioides puede convivir con ambas. Por consiguiente, el bloqueo de receptores opioides disminuiría la recompensa, lo que puede modificar el deseo de consumir alcohol. En este sentido, los estudios realizados con diversos antagonistas opiáceos, como naloxona, naltrexona o nalmefene, ponen de manifiesto, de manera estable, que estos agentes disminuyen el consumo de alcohol, tanto en ratas como en monos, en especial en los que exhiben una alta preferencia por el alcohol (Volpicelli y cols., 1997).

Además, la naltrexona es un tratamiento aprobado para la prevención de las recaídas en pacientes alcohólicos. En efecto, múltiples estudios indican la existencia de una asociación entre consumo de alcohol y liberación de opioides endógenos que participarían en las propiedades reforzadoras del alcohol. De hecho, la naltrexona reduce en un $50 \%$ la tasa de recaídas y los individuos permanecen más tiempo en abstinencia. Además, cuando el sujeto realiza una consumición inicial de alcohol, la naltrexona impide que se caiga en un consumo masivo, al permitir que el individuo no pierda el control (Guardia, 1998).

Desde una perspectiva clínica, Garbutt y cols. (1999) han realizado un reciente trabajo "basado en la evidencia" sobre la eficacia de los tratamientos farmacológicos de la dependencia al alcohol. En el citado trabajo se señala que los 3 ensayos clínicos evaluados con naltrexona, que incluyen un modesto número de pacientes ( $n=271$ ), están realizados con una adecuada metodología y aportan suficientes datos para evaluar su eficacia, siendo los resultados con naltrexona superiores a los obtenidos con el placebo. En este sentido, la naltrexona redujo la tasa de recaídas. Además, la naltrexona parece tener su efecto primario en su capacidad de reducir la respuesta emocional del paciente al consumo de alcohol. Así, un consumo ocasional de alcohol no se acompaña de una recaída grave. Por tanto, el tratamiento con naltrexona parece no impedir la bebida, pero una vez que ésta se produce el paciente no encuentra los efectos reforzadores esperados y evita el consumo descontrolado. En este sentido, pueden existir diferencias entre pacientes. Así, algunos consideran el producto como una gran ayuda para evitar recaídas, en especial los que mejor cumplimentan el tratamiento, mientras que otros no obtienen beneficios. Estudios en curso, realizados en amplias poblaciones de alcohólicos, darán mejor información de la trascendencia de este fármaco en la clí- 
FIGURA 3: Mecanismo de interacción postulado para explicar la relación entre el sistema opioide y el consumo de alcohol.

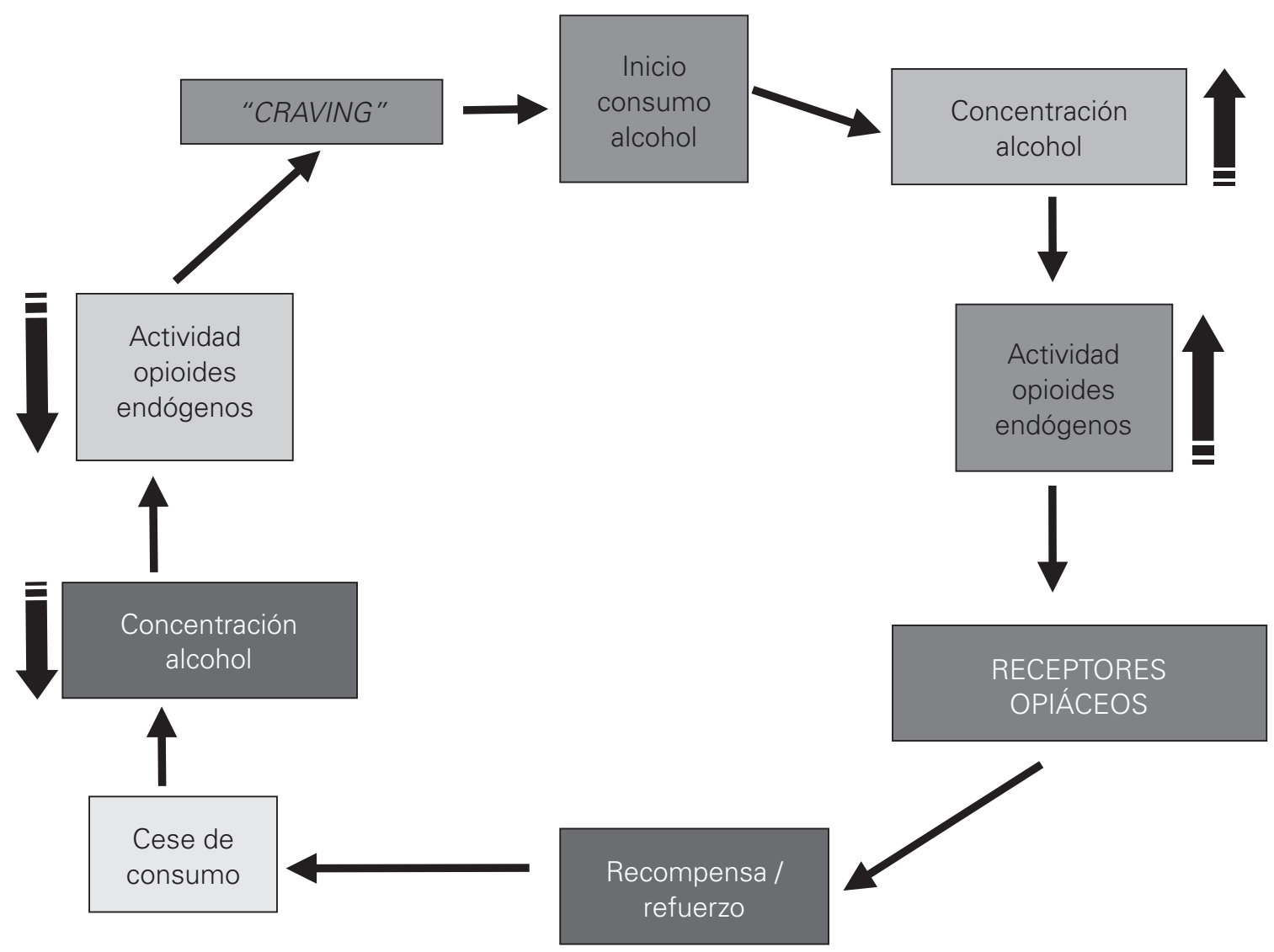

nica diaria (Garbutt y cols., 1999). Por el momento, se puede concluir que la naltrexona disminuye la tasa de recaídas, los días de consumo y la frecuencia de episodios de consumo exagerado. Se postula que estos efectos son consecuencia de un efecto "anticraving", por el que se evita la pérdida de control. El efecto preventivo de recaídas de naltrexona es manifiesto en presencia de otras medidas psicosociales, lo que pone de manifiesto, una vez más, la necesidad de realizar un abordaje terapéutico multifactorial de las dependencias.

El nalmefene es un antagonista opiáceo, estructuralmente emparentado con la naltrexona, pero con una mayor afinidad por el bloqueo de receptores opiáceos $m$, d y k. Los resultados de un primer estudio piloto fueron prometedores, observándose una menor tasa de recaídas en los 8 sujetos que completaron el estudio con la dosis de $40 \mathrm{mg} /$ día, con respecto a la dosis de 10 mg/día y a placebo (Mason y cols., 1994). Un estudio posterior, realizado por el mismo grupo (Mason y cols., 1999), en alcohólicos de alto consumo y tratamiento con nalmefene a las dosis de 20 y 80 $\mathrm{mg} /$ día, puso de manifiesto que ambas dosis no eran diferentes entre sí, pero si eran superiores al placebo en la reducción de las recaídas graves. Estos datos hablan a favor de la importancia del bloqueo de receptores opioides en la prevención de recaídas severas en los alcohólicos.

Desde una perspectiva farmacológica, resulta interesante explorar mecanismos diferentes a los opioides en la dependencia al alcohol. En este sentido, el acamprosato (acetilhomotaurinato cálcico) constituye una buena alternativa. En un principio se pensó que el acamprosato estimulaba la neurotransmisión inhibitoria mediada por el GABA (Sass y cols., 1996), hecho que posteriormente se ha descartado. En la actualidad, se sabe que el mecanismo de acción del acamprosato se basa en su capacidad de inhibir la transmisión de aminoácidos excitatorios, en especial el glutamato y el n-metil-d-aspartato (NMDA), inhibiendo la excitabilidad neuronal postsináptica. Además, el acamprosato interactúa con los canales de calcio voltaje dependientes, inhibiendo la regulación al alza de estos canales provocada por el síndrome de abstinencia a alcohol en la rata (Spanagel y Zieglgänsberger, 1997). 
Desde el punto de vista clínico, el acamprosato ha sido estudiado en diversos ensayos que incluyen un número de pacientes superior a los estudios realizados, hasta el momento, con naltrexona. Los datos recogidos son suficientes para confirmar que el acamprosato exhibe una eficacia superior a la del placebo. Del conjunto de estos estudios se puede concluir que el acamprosato reduce la frecuencia de bebida y existen evidencias que indican que potencia la abstinencia (Garbutt y cols., 1999). Así, el acamprosato, con respecto al placebo, aumenta la tasa de abstinencia continuada al alcohol, la duración de la misma y alarga el tiempo de inicio de la primera toma de alcohol. Asimismo, el acamprosato aumenta el número de días sin consumo de alcohol a lo largo de un año, viéndose este efecto potenciado por la asociación con disulfiram (Hoes, 1999).

El papel de agentes moduladores de la transmisión serotoninérgica central ha sido también evaluado en la prevención de recaídas. Existen múltiples líneas de evidencia que indican la existencia de una disfunción serotoninérgica en la dependencia al alcohol (Heinz y cols., 1998), así como en el fenómeno del craving y en las recaídas (Ciccocioppo, 1999). Así, una deficiencia en el funcionalismo serotoninérgico, que puede afectar a los mecanismos del control de los impulsos, puede contribuir a la perdida de control de la bebida y llevar a una recaída. Asimismo, se ha podido constatar una significativa reducción del transportador de serotonina en tronco cerebral de alcohólicos, guardando este hecho una correlación con el tiempo de duración del alcoholismo y con la sintomatología depresiva y ansiosa durante la abstinencia (Heinz y cols., 1998). Por otra parte, basándose en esta disfunción serotoninérgica, se ha acumulado una variada experiencia clínica con agentes que potencian el funcionalismo serotoninérgico, como la buspirona, el ondansetron y, especialmente, los inhibidores selectivos de la recaptación de serotonina (ISRS), en la reducción de la ingesta de alcohol. En la mayoría de estos estudios con potenciadores del funcionalismo serotoninérgico, la presencia de alteraciones del humor era relativamente frecuente. De los diferentes estudios clínicos realizados parece poder concluirse que no existen evidencias para el tratamiento del alcoholismo primario con estos agentes. Sin embargo, algunos ISRS, como fluoxetina o citalopram, pueden ser de ayuda en la prevención de recaídas, en especial en pacientes con patología psiquiátrica, ansiedad y/o depresión comórbida. Por otra parte, la fluoxetina parece incrementar la eficacia de la naltrexona en la prevención de recaídas a la heroína, así como al alcohol, probablemente contribuyendo a disminuir la avidez y el ansia por la droga y la disforia que provoca en algunos pacientes la naltrexona. Por el contrario, la buspirona, incluso en pacientes con ansiedad asociada, aporta resultados menos consistentes (Garbutt y cols., 1999).

\section{MECANISMO DE ACCIÓN DE LOS AGENTES "ANTICRAVING"}

Un tema de gran interés es el conocimiento de los mecanismos por los que algunos agentes presentan eficacia "anticraving". Tanto en el caso de la naltrexona como en el del acamprosato, se ha sugerido que la eficacia de estos agentes refleja la producción de cambios en los factores del entorno (cue), relacionados con la toma de alcohol. En este sentido, las señales externas, así como las internas (cambios en los estados del humor), relacionadas con la toma de alcohol, inducen cambios físicos y psíquicos en el sujeto, que condicionan tanto el refuerzo como la abstinencia. En situaciones experimentales en las que se administra alcohol, estas señales (cue) pueden incluso modificar ciertos efectos del alcohol, tales como la tolerancia. Mayor importancia tiene la consideración de que estas señales permanecen activas durante periodos muy prolongados de abstinencia. El examen de estas señales condicionadas, tanto en el animal de experimentación como en el humano, ha sido propuestos para dilucidar la naturaleza del craving.

En relación con la naltrexona, podría postularse que, al bloquear los receptores opiáceos, impediría el refuerzo provocado por la liberación de opioides endógenos inducidos por el alcohol (Fig. 4). Se podría especular que, en las señales del entorno, relevantes para que se produzca el craving, estén implicados los opioides endógenos y, en esta línea, la naltrexona evitaría el craving y otros efectos del cue, al bloquear la activación de los sistemas de opioides endógenos. En numerosos estudios se ha podido comprobar que la administración de alcohol, de forma crónica, produce cambios en los niveles de opioides endógenos, en sus receptores, así como en los niveles de RNAm de los péptidos opioides (Herz, 1997). Estas modificaciones pueden estar en la base del craving y son motivadas por cambios neuroadaptativos, consecuencia de variaciones en la expresión génica del sistema opioide, provocados por el alcohol, con el fin de reaccionar, de una manera plástica, a los estímulos o señales del entorno. En este sentido, es conocido que algunos de los cambios provocados, a largo plazo, en los sistemas comentados, tienen su punto de inicio en eventos genéticos provocados por la transactivación de genes de respuesta temprana. Resulta destacable la capacidad de los péptidos opioides de influir, a través de un mecanismo reversible por naltrexona, en la expresión de estos genes, en varios grupos de neuronas centrales, y la capacidad de la proteína c-fos de transactivar a los genes codificadores de los péptidos opioides (Spanagel y Zieglgänsberger, 1997).

Por su parte, el acamprosato puede ejercer su acción anticraving mediante la prevención de las respuestas condicionadas a la abstinencia de alcohol (Fig. 4), ya que inhibe muchos de los síntomas de la absti- 


\section{FIGURA 4: Mecanismo de acción de los agentes "anticraving". Acción hipotética de la naltrexona y el acamprosato sobre los posibles mecanismos implicados en el consumo de alcohol.}

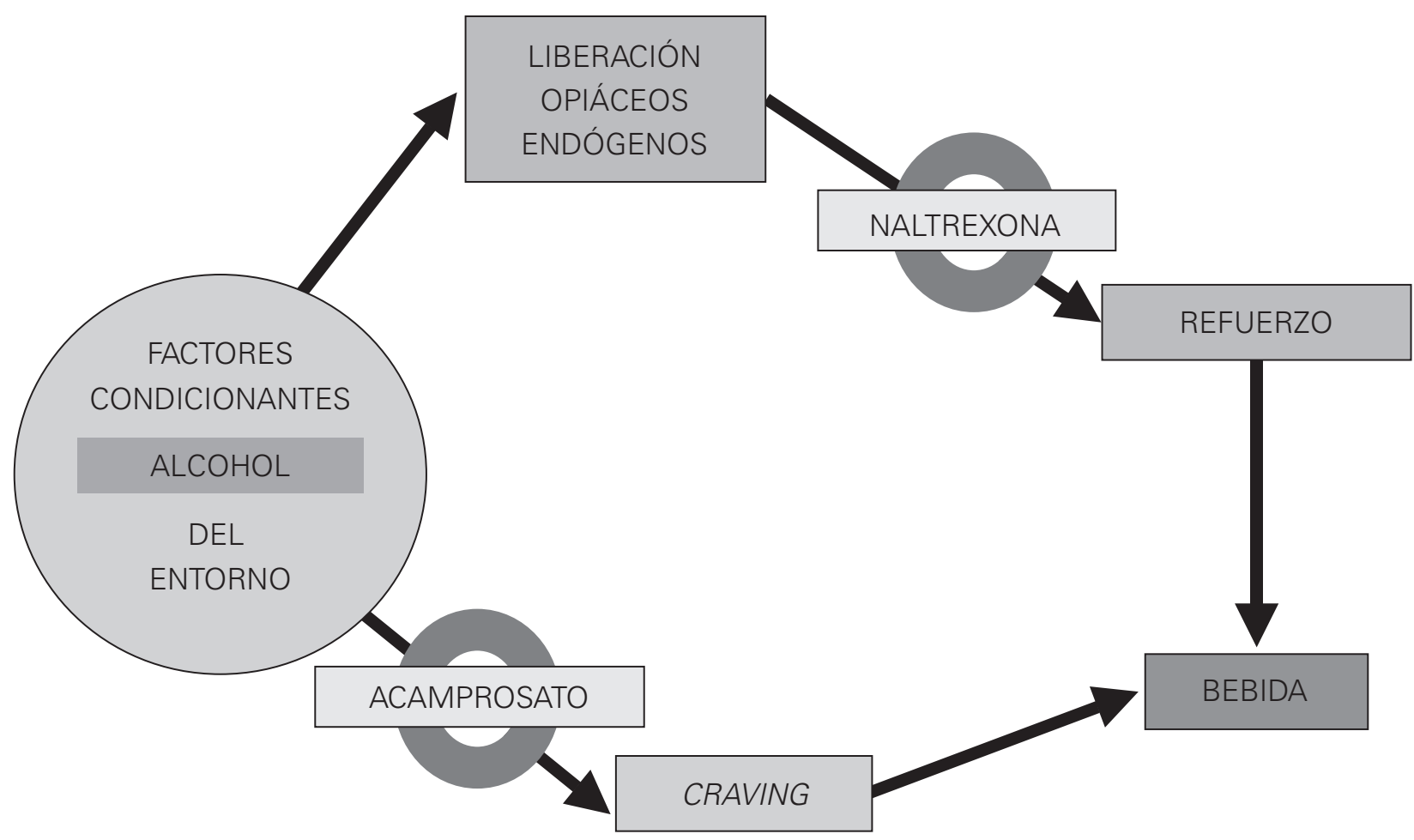

nencia aguda al alcohol, que se acompañan de hiperexcitabilidad neuronal. Estas modificaciones podrían estar relacionadas con los cambios en las distintas subunidades de los receptores de NMDA motivados por la ingesta crónica de alcohol (Nutt, 1996). Además, los canales de calcio voltaje dependientes están fuertemente implicados en la hiperexcitabilidad inducida por la abstinencia al alcohol. En este sentido, el acamprosato, reduciendo la actividad de estos canales, disminuiría la hiperexcitabilidad neuronal observada durante la abstinencia. Por tanto, el acamprosato centraría su acción anticraving en provocar un contrapeso de los cambios en receptores NMDA y canales de calcio, lo que provocaría una reducción de la hiperexcitabilidad neuronal, inducida por las respuestas condicionadas relacionadas con la abstinencia.

En definitiva, los mecanismos implicados en el efecto protector de las recaídas alcohólicas por parte de naltrexona y acamprosato serían diferentes (Fig. 4). La naltrexona interfiere el refuerzo positivo provocado por la liberación de opioides endógenos, al bloquear sus receptores, y antagonizaría los estímulos condicionados relacionados con dicho refuerzo positivo. En este sentido, es necesario dilucidar si, en el humano, la naltrexona previene las recaídas de forma exclusiva por un bloqueo del refuerzo positivo del alcohol o si, además, interviene un efecto anticraving (Nutt, 1996; Spanagel y Zieglgänsberger, 1997). Por su parte, el acamprosato parece actuar predominantemente inhibiendo la hiperexcitabilidad neuronal asociada a la abstinencia y, además, inhibiendo la reactividad inducida por las señales (cue) condicionadas relacionadas con la misma (Nutt, 1996; Spanagel y Zieglgänsberger, 1997).

\section{CONCLUSIÓN}

El estudio del papel de las recaídas en la dependencia a sustancias ha sido motivo de múltiples investigaciones. Los modelos animales tienen utilidad, pero resulta arriesgado, si no erróneo, extrapolar directamente sus resultados a los seres humanos. En éstos, las variables son mucho más numerosas (tipos de pacientes, cantidades de consumo, tipos de alcoholismo, polidrogodependencia, patología dual, comorbilidad somática, cumplimentación, duración del seguimiento, expectativas del paciente, tratamiento psicosocial, etc.) (Regier y cols., 1990), y, por ende, los resultados rara vez se pueden aplicar de forma universal. 
La investigación farmacológica y clínica debe avanzar en el mejor conocimiento del craving y los cue como elementos importantes en las recaídas, pero considerándolos sólo como una pieza más del problema. Los componentes emocionales, sociales, familiares, económicos y la vida de relación, son otras piezas de la dependencia que requieren un abordaje terapéutico a la medida de cada paciente. El tratamiento del paciente dependiente de sustancias no es un proceso sencillo, sino que, como sucede con cualquier otra patología crónica, es largo y entraña dificultades. Las herramientas farmacológicas pueden ser de gran ayuda, pero nunca deben conformarse como un abordaje único y aislado. Conseguir que un paciente invalidado por el abuso de sustancias se reintegre a un funcionamiento normal, dentro de la sociedad, justifica que empleemos todos los medios a nuestro alcance para conseguir el éxito terapéutico.

\section{BIBLIOGRAFIA}

Alamo, C. y López-Muñoz, F. (1996). Aspectos neurofarmacológicos de la dependencia a opiáceos. En A. Valbuena y C. Alamo. Avances en toxicomanías y alcoholismo. Aspectos conceptuales, farmacológicos, clínico terapéuticos y médico-legales. Alcalá de Henares. Servicio de Publicaciones de la Universidad de Alcalá, 39-56.

Alamo, C., López-Muñoz, F. y Cuenca, E. (1999). Bases neurofarmacológicas para el abordaje terapéutico de la patología dual. En R. Salvanés y C. Alamo. Avances en patología dual. Aspectos diagnósticos, clínicos, terapéuticos y asistenciales. Alcalá de Henares. Servicio de Publicaciones de la Universidad de Alcalá, 163-190.

Alguacil, L.F., Iglesias, V. y Álamo, C. (1991). Mecanismos bioquímicos de la dependencia a opiáceos. Farmacología del SNC, 5, 17-22.

Altman, J., Everitt, B.J., Glautier, S. et al. (1996). The biological, social and clinical bases of drug addiction: comentary and debate. Psychopharmacology, 125, 285-245.

American Psychiatric Association (1995). Practice guidelines for the treatment of patients with substance use disorders: alcohol, cocaine, opioids. American Journal of Psychiatry, 152, 4-59.

Baker, T.B., Morse, E. y Sherman, J.E. (1987). The motivation to use drugs: a psychobiological analysis of urges. En Rivers, P.C. The Nebraska Symposium on Motivation: Alcohol Use and Abuse. Lincoln. University of Nebraska Press, 257-323.

Best, S.E., Oliveto, A.H. y Kosten, T.R. (1996). Opioid addiction. Recent advances in detosification and maintenance therapy. CNS Drugs, 6, 301-314.
Cabrera-Forneiro, J. (1998). Introducción general. En Manual de drogodependencias. Madrid, Cauce Editorial, 13-28.

Chick,J., Gough,K., Falkowski,W. et al.(1992). Disulfiram treatment of alcoholism. British Journal of Psychiatry, 161, 84-89.

Ciccocioppo, R. (1999). The role of serotonin in craving: from basic research to human studies. Alcohol and Alcoholism, 34, 244-253.

Davis, R.M., Wagner, E.G. y Groves, T. (2000). Advances in managing chronic disease. British Medical Journal, 320, 525-526.

Foltin, R.W. y Fischman, M.W. (1994). Effects of buprenorphine on the self administration of cocaine by humans. Behavioural Pharmacology, 5, 79-89.

Garbutt, J.C., West, S.L., Carey, T.S. et al. (1999). Pharmacological treatment of alcohol dependence. A review of the evidence. JAMA, 281, 1318-1325.

Gerada, C. y Ashworth, M. (1997). ABC of mental health: Addiction and dependence-I llicit drugs. British Medical Journal, 315, 297-300.

Gianoulakis, C., Angelogianni, P., Meaney, M. et al. (1990). Endorphins in individuals with high and low risk for development of alcoholism. En Reid, L.D. Opioids, Bulimia, and Alcohol Abuse and Alcoholism. New York, SpringerVerlag, 229-246.

Guardia, J. (1998). Naltrexona en el tratamiento de la dependencia del alcohol. Psiquiatría Biológica, 5, 65-74.

Heinz, A., Ragan, P, Jones, D. W. et al. (1998). Reduced central serotonin transporters in alcoholism. American Journal of Psychiatry, 155, 1544-1549.

Herz, A. (1997). Endogenous opioid systems and alcohol addiction. Psychopharmacology, 129, 99111.

Hoes, M. (1999). Relapse prevention in alcoholics. A review of acamprosate versus naltrexone. Clinical Drug Investigation. 17, 211-216.

Iglesias, V., Alguacil, L.F., Alamo, C. y Cuenca, E. (1992). Effects of yohimbine on morphine analgesia and physical dependence in the rat. European Journal of Pharmacology. 211, 35-38.

Kosten, T. (1998). Addiction as a brain disease (editorial). American Journal of Psychiatry, 155, 711713.

Leshner, A.I. (1999). Science-based views of drug addiction and its treatment. JAMA, 282, 13141316.

Marwick, C. (1999). Ilicit drug users not idle; report says $70 \%$ go to work. JAMA, 282, 1-3.

Mason, B.J., Ritvo, E.C., Morgan, R.O. et al. (1994). A double blind placebo controlled pilot study to evaluate the efficacy and safety of oral nalmefene 
HCL for alcohol dependence. Alcoholism Clinical and Experimental Research, 18, 1162-1167.

Mason, B.J., Salvato, F.R., Williams, L.D. et al. (1999). A double blind placebo controlled study of oral nalmefene for alcohol dependence. Archives of General Psychiatry, 56, 719-724.

Nestler, E.J. (1994). Molecular neurobiology of drug addiction. Neuropsychopharmacology, 11, 77-87.

National Institute of Health (1998). Effective medical treatment of opiate addiction. $\mathrm{NIH}$ Consens Statement. JAMA, 280, 1936-1943.

Nuñez, A., Soto, C. y Pastor, A. (1998). Consumo de drogas en España: reflexiones sobre sus tendencias. Psiquiatría Pública, 10, 355-360.

Nutt, D.J. (1996). Addiction: brain mechanisms and their treatment implications. The Lancet, 347, 31-36.

O'Brien, C.P. (1997). Progress in the science of addiction. American Journal of Psychiatry, 154, 1195-1197.

O'Brien, C.P. y McLellan, A.T. (1996). Mitos sobre el tratamiento de la adicción. The Lancet (Ed. Esp.), 347, 237-240.

Ochoa, E. (1998). Naltrexona en el tratamiento de la dependencia del alcohol. Actas Luso-Españolas de Neurología, Psiquiatría y Ciencias Afines, 26, 51-57.

Regier, D.A., Farmer, M.E., Rae, D.S. et al. (1990). Comorbidity of mental disorders with alcohol and other drug abuse. Results from the Epidemiological Catchment Area Study. JAMA, 264, 2511-2818.

Rubio, G., Alguacil, L.F., Alamo, C., et al. (1992). Relapse to opiate provoques biphasic changes of blood pressure in heroin-withdrawn addicts treated with clonidine. Drug and Alcohol Dependence, 30, 193198.

Sass, H., Soyka, M., Mann, K. y Zieglgänsberger, W. (1996). Relapse prevention by acamprosate.
Results from placebo-controlled study on alcohol dependence. Archives of General Psychiatry, 53, 673-680.

Spanagel, R. y Zieglgänsberger, W. (1997). Anticraving compounds for ethanol: new pharmacological tools to study addictiv processes. Trends in Pharmacological Sciences, 18, 54-59.

Self, D.W. y Eric, J.N. (1995). Molecular mechanisms of drug reinforcement and adicction. Annual Review of Neuroscience, 18, 463-495.

Tiffany, S.T. (1990). A cognitive modell of drug urges and drug-use behavior: role of automatic and nonautomatic processes. Psychological Review, 97, 147-168.

Ulm, R.R., Volpicelli, J.R. y Volpicelli, L.A. (1995). Opiates and alcohol self-administration in animals. Journal of Clinical Psychiatry, 56, 5-14.

Volpicelli, J.R., Rhines, K.C., Rhines, J.S. et al. (1997). Naltrexone and alcohol dependence. Role of subject compliance. Archives of General Psychiatry, 54, 737-742

Weaver, M.F., Jarvis, M.A.E. y Schnoll, S.H. (1999). Role of the primary care physician in problems of substance abuse. Archives of Internal Medicine, 159, 913-924.

West, S.L. (1999). Pharmacological treatment of alcohol dependence. JAMA, 281, 1318-1325.

Wikler, A. (1973). Dynamics of drug dependence: implications of a conditioning theory for research and treatment. Archives of General Psychiatry, 28, 611-616.

Widnell, K.L., Self, D.W., Lane, S.B., et al. (1996). Regulation of CREB expression: In vivo evidence for a functional role in morphine action in the nucleus accumbens. Journal of Pharmacology and ExperimentalTherapy, 276, 306-315. 
